\title{
DETERMINATION OF THE INFORMATIONAL CONTENT OF SYMPTOMS IN THE DYNAMIC PROCESSES OF ASSESSING THE PATIENT'S CONDITION IN E-HEALTH
}

\author{
Yaroslav Shevchenko \\ Department of Medical Informatics \\ Shupyk National Healthcare University of Ukraine \\ 9 Dorohozhytska str., Kyiv, Ukraine, 04112 \\ misterMishka@ukr.net
}

\begin{abstract}
The study is devoted to substantiating the tactics of choosing the signs of the patient's condition for diagnostic decision-making on corrective medical intervention in mobile medicine.

The aim of the research: to study a creation of a methodology for determining the integral informativeness of the patient's symptoms during remote monitoring of his condition.

Materials and methods: this article is based on search results in PubMed, Scopus, MEDLINE, EMBASE, PsycINFO, Global Health, Web of Science, Cochrane Library, UK NHS HTA articles published between January 1991 and January 2021 and containing the search terms «information technology», «Mobile medicine», «digital pathology» and «deep learning», as well as the results of the authors' own research. The authors independently extracted data on concealment of distribution, consistency of distribution, blindness, completeness of follow-up, and interventions.

Results: concluded that to determine the Informativeness of symptoms in mobile monitoring of patients, it is possible to use risk indicators of predicted conditions as a universal method. Given that the Informativeness of the patient's condition changes constantly, for online diagnosis of conditions during remote monitoring of the patient it is recommended to use the function of informative symptoms from time to time and use a set of approaches to assess the Informativeness of patient symptoms. It is proposed to use the strategy of diagnosis and treatment using probabilistic algorithms based on the values of the risk of complications of the pathological process, as well as the formulas of Kulbach and Shannon to determine individual trends in the pathological patient process.

Conclusion: there was proposed to use risk indicators of predicted conditions as a universal method for determining the informational content of symptoms in mobile monitoring of patients.
\end{abstract}

Keywords: clinical signs, information technologies, mobile medicine, digital pathology, patient care.

DOI: $10.21303 / 2504-5679.2021 .001976$

\section{Introduction}

Modern care of patients becomes more individualized. Personalization and constant monitoring of condition of patients is characterized by constant monitoring of the patient's condition, its quantitative assessment, forecasting the values of vital physiological parameters, etc. Hence the rapid development of e-health [1]. Remote patient monitoring systems are designed to obtain a number of physiological data from patients. Herewith, a characteristic feature of the current period of development of e-health technologies is that in the tasks of designing new devices appear tasks not only of monitoring, but also algorithms for making decisions about treatment [2]. At the same time, taking a decision to clarify the diagnosis put forward a number of new requirements for decision-making logic, new risks associated with the evaluation of information from remote e-health devices, especially if you have to deal with chronic diseases [3].

If we analyze the problems with the evaluation of information, then, first of all, we have to face the problem of dimensionality. Therefore, the problem of reducing the dimensionality of the space of attributes and highlighting the most informative among them is especially topical for medical practice. As a result, the problem of selection of informative features can be formulated. It can be considered in the format of a combinatorial optimization problem [4]. But, with the assessment of the informativeness of the information obtained is not so simple. As a matter of fact, namely the informational content determines the strategy of diagnosis and treatment of the patient. Indeed, the doctor, who is not able to provide a comprehensive patient workup, besides the fact that 
it is necessary to know the validity of the symptoms, it is also important to assess whether the information received is enough to make a diagnosis, and if it is not enough, which examinations will provide the doctor in the shortest time necessary information for further examination [5]. To date, the assessment of the informational content of the patient's condition indicators obtained during remote monitoring is relatively unexplored in terms of informativeness and validity of the patient's condition during their continuous monitoring [6].

The aim of the research. To explore the methodology for determining the integral informational content of the patient's symptoms during remote monitoring of his condition.

\section{Materials and methods}

We searched the following electronic bibliographic databases MEDLINE, EMBASE, PsycINFO, Global Health, The Cochrane Library (Cochrane Database of Systematic Reviews, Cochrane Central Registry of Controlled Tests [CENTRAL], Cochrane Methodological Register), Health Technology Assessment Database and the Internet Science (cited index of natural and social sciences) for articles published between January 1991 and January 2021 containing searches for «information technology», «mobile medicine», «digital pathology» and «deep learning», and also the results of the authors' own research. Lists of links also included studies. A list of subheadings (MeSH) and text words used in the search strategy can be found in text S1 [7]. All of these terms have been combined with the Cochrane Library MEDLINE filter for controlled intervention testing. We only included studies in which a mobile electronic device was a specific intervention assessed, i. e., a control group that receives only paper information [7].

\section{Result}

\subsection{Definition and general formulation of the problem}

Let us understand by informational content the meaning of information for solving a specific problem [8] states in medical diagnostics, predicting the consequences of treatment, theoretical and practical results of the methods and values of indicators of the informational content of clinical signs are studied in detail.

The greatest difficulty in assessing the patient's condition is the assessment of the informational content of the information obtained. Usually, a measure of the value of the symptom is considered to diagnose a particular disease $D j$ or the patient's condition [9]. The symptom can be quite useful for diagnosing some diseases and useless for others [9]. To determine the role of a symptom in a class of phenomena, an integral measure is clearly needed - the overall diagnostic value of the symptom. The measure of the diagnostic value of a symptom for a class of different conditions of the patient should be equal to the mathematical expectation of the measures of the diagnostic value of the symptom for individual conditions [10]. But, the informational content of the research depends on its place in the diagnostic process [11]. This dependence «acts» due to the probability of diagnoses calculated on the basis of previous researches. The same probabilities serve a priori to calculate the possibilities of diagnoses after receiving the results of the next examination [12]. Therefore, it is desirable to use estimates of informational content that do not depend on the a priori distribution [13].

Mathematical software for calculating of informational content has been developed for the last 50 years [12]. It has become quite obvious that the informational content of symptoms and clinical signs depends on a large number of factors. Among them should be mentioned the presence of comorbidities, genetic and other clinical signs with which this symptom has a possible pathogenetic link, medical intervention and etc. The stage of diagnosis, the place of symptoms in the diagnostic process, as well as the dynamics of the pathological process have a significant impact on the amount of informational content of symptoms [14]. In other words, the concept of «informational content of signs» works in the space of strictly defined clusters of states, at specific stages of the processes of recognition and prediction of pathological processes. These features of the value of informational content have been considered in many studies [8]. As a result, it can be concluded that for the three main processes of modern medical practice (verification of condition of patients; identification of the existing state of existing clusters; individualization of the approach to correct patients' condition) should be developed new or attributed to them previously developed devices [15]. 


\subsection{Processes of medicine's transformation. Information technologies in practical medicine}

Information technology (IT) is spreading with incredible speed in practical medicine. Since 2000 in publications shown that new IT significantly improves the quality of medical care [9]. Robotics, computer monitoring supervision of the patient, augmented reality systems, the Internet, mobile medicine, telemedicine, and simulation medicine have found widespread implementation. Already today, «artificial intelligence» (AI) is working in medicine, which after the first successful application in medicine $[4,16]$ has become a popular and important direction in both researches and practical medicine. Devices have been developed to improve the quality of life of people with Alzheimer's disease [17], in oncology and other areas [18]. We emphasize that if according to statistics, in USA hospitals, the accuracy of the appointment of optimal treatment after diagnosis of lung cancer is about $50 \%$, then the use of AI, these figures reach $90 \%$. The method of treatment can be adjusted depending on changing situations [19].

The development of information technology has provided a new approach for providing medical care, it is called integrated (intramural) medicine [20]. It is considered as a response to the fragmentary providing of medical and social services, which is a generally recognized problem in many health care systems [21]. According to the World Health Organization, complex medical care is a concept that joins together resources, providing, management and organization of services related to diagnosis, treatment, care, rehabilitation and health promotion [22].

Another area related to the development and implementation of information technology, which can lead to major changes in the organization of providing of medical services to the population is called patient participation [23]. The emergence of this area is due to the fact that the traditional paternalistic model of medicine, in which patients had a small voice in communication with the doctor about their health, slowly, but correctly evolved towards a model in which patients and doctors work in partnership to achieve the common goal is to improve health [24, 25]. This can be crucial in dramatically improving the quality of providing medical care to ensure that the patient is involved in the health decision-making process $[26,13]$. However, the emphasis on the decision-making process does not include the many and varied areas of medical support in which the patient could participate [18].

Today robotics is becoming reality, making possible to move to the next stage of human evolution. The new paradigm is to implement a crucial global transition from the use of sensor network technology to the network of actuators. This paradigm marks the emergence of a fundamentally new approach, because it creates a ubiquitous, intelligent, «live» Internet $[14,26]$. It becomes possible to provide the logic of determining trends, the real risks of pathology to «conventional devices», and in the near future - to make decisions without human intervention, especially in critical situations [3]. All this led to the emergence of the Internet of Things - one of the most popular scientific ideas of modern computer science, which is now being actively implemented [17, 27$]$.

The Internet of Things is a global network of connected to the Internet of Things devices equipped with sensors, sensing devices, and signalling devices, and the Internet of Medical Things is a combination of medical devices, software applications, and healthcare systems and services [18]. This infrastructure aims to increase the availability of medical care while improving patient health and satisfaction with health services [8]. Digital devices can use special sensors to receive various signals from the outside world, interact with other devices, exchange data for remote monitoring of the condition of objects, analyze the collected data and make decisions based on them [29, 30]. Examples of health care include remote monitoring of patients' health using a variety of sensors: video cameras, temperature sensors, blood pressure, etc. The interaction of the devices is provided in such a way that from now on long-term data storage becomes possible [31, 16].

At the same time, the development and implementation of IT has created a new list of problems that are subject to immediate resolution [32].

The pandemic of coronavirus infection (COVID-19), caused by the SARS-CoV-2 virus, has led to significant restrictions on regular medical treatments aimed at maintaining health, and has created challenges for the work of medical institutions and diagnostic laboratories [13].

Treatment of patients becomes individualized. Personalization and constant monitoring of patients determines the new «environment of their existence», which is characterized by constant 
monitoring of the patient's condition, its quantitative assessment, forecasting the values of vital physiological parameters [33]. Accordingly, mobile medicine began to develop rapidly. There is a large number of microelectronic technologies designed for remote monitoring of patients' condition [34]. Remote patients' monitoring systems are designed to obtain a number of physiological data from patients. The most common data are electrocardiogram (ECG), electroencephalogram (EEG), heart rate and respiratory rate, blood oxygen volume or pulse oximeter, nervous system signals, blood pressure, body/skin temperature and blood glucose level [2, 35]. In addition, data on the patient's weight, activity levels and sleep status are sometimes collected. A characteristic feature of the current period of development of microelectronic technologies is that in the tasks of designing new devices there are tasks not only of monitoring, but also algorithms for making decisions about treatment $[23,6]$. At the same time, taking the decision to clarify the diagnosis or ordering treatment has put forward a number of new requirements for the logic of decision-making, new risks associated with the evaluation of information from remote devices, and etc. It is clear that in a special risk zone began to be elderly people suffering from chronic diseases [14].

If we analyze the problems on evaluation of information, then, first of all, we have to face the problem of dimensionality. The number of different signs that describe the patient's health condition is very large and sometimes reaches several tens and hundreds of indicators [36, 37]. Therefore, the problem of reducing the dimensionality of the space of signs and highlighting the most informative among them is especially relevant for medical practice. As a result, the problem of selection of informative features can be formulated. It can be considered in the format of a combinatorial optimization problem. But the greatest difficulty in assessing the patient's condition is the assessment of the informativeness of the information obtained [1]. Basically, the informativeness determines the strategy of diagnosis and treatment of the patient. Indeed, for a physician who is unable to provide a comprehensive examination of the patient, in addition that it is necessary to know the validity of the symptoms, it is also important to assess whether the information obtained is sufficient to make a diagnosis and, if not enough, what research (shortest for time period) provide the physician with the necessary information for further examination. The same considerations apply to decision-making regarding the tactics of medical intervention [22].

\subsection{Identification of patient's conditions in mobile medicine}

Mobile medicine (m-Health) should provide medical care and control of a healthy lifestyle using wireless telecommunications technology and mobile devices [35]. It began to spread at the end of the last century [5], but the meaning of the term is constantly changing and expanding: from simple healthcare practices using the Internet to the use of complex systems for assessing the state of the body.

In most technologies, mobile medicine is based on the use of mobile devices designed to monitor certain vital parameters, process, store and transmit this data. Among mobile personal devices, a mobile phone (smartphone) is most often used, because a person almost always carries it with him; it can store a complete medical record of the user in its memory, which can be vital at critical moments [14].

Almost all the flagship smartphones of the largest manufacturers contain sensors and software for m-Health (pedometer, pulse oximeter, health diaries and many more). Mobile applications help to follow a dietary regime and medication. A smartphone can also become a personal trainer that distributes workloads wisely and makes progress systematically with the user [29].

However, without a correct interpretation, all this information is meaningless. Moreover, there are already technologies that are ready to provide ample opportunities for practical medicine, but which the conservative medical community is just beginning to considerate as a convenient tool for working with patients. The main problem that delays the implementation in practice is the logical interpretation of the information obtained by the doctor, because without professional opinion, any data is simply an array of disordered information [13].

At the same time, it is extremely important to emphasize that mobile medicine can bring unexpected and important results today. Let us analyze more deeply, in particular, the evaluation of the effectiveness of remote interaction between doctor and patient [30]. 
A study, conducted by team of Scheper Hospital in the Netherlands included telemonitoring of 102 patients with chronic cardiac failure [8]. The set of measures included not only monitoring of cardiac activity, but also distance education courses, various exercises and control of medication. If at the beginning of the study 102 patients had 132 emergency hospitalizations per year (1.29 per patient), then after a year of remote monitoring their number decreased to 32 ( 0.31 per patient), or $76 \%$. The total number of bed-days for all patients due to all hospitalizations decreased from 975 to 662 per year (by $32 \%$ ) [8].

In the second study, a group of authors from the USA and Canada [11] analyzed publications and surveys about evaluation of the effectiveness of remote monitoring of patients with chronic cardiac failure. According to the analysis of 15 surveys published in the period from 2003 to 2015, there was a decrease in the relative risk of overall mortality (range 0.60-0.85) and hospitalization due to cardiac failure (0.64-0.86) compared with control. The absolute reduction of similar risks varied in the values of $1.4 \%-6.5 \%$ and $3.7 \%-8.2 \%$, respectively [26].

Finally, we pay our attention on the study by a team of researchers led by Y. Nohara [6], who conducted a mass screening of the health condition of 16,741 residents of Bangladesh cities and villages. All patients according to the results of anthropometric and clinical data were divided into 4 risk groups according to condition. Patients at high risk received teleconsultations and doctor's appointments. The study used a tablet PC, tonometer, glucometer, test strips for urine tests and other portable equipment. The main criterion for the effectiveness of screening, the authors considered a lowering the average arterial blood pressure. As a result of monitoring, most patients showed improvement of condition, which allowed them to be transferred to lower risk groups, and the average level of systolic arterial blood pressure decreased from 121 to 116 millimetres of mercury [37].

Another important practical consequence of mobile medicine is the possibility of involving narrowly specialized medical specialists in the diagnosis and treatment of patients [4], which can be difficult for most regions of the country. Mobile medicine contributes to the effective solution of dispensary observation programs for patients with chronic diseases, especially in cases of a large number of patients who need dynamic monitoring [38].

Most studies focus on the possibility of achieving a significant economic effect when using mobile medicine. It is clear that prevention and treatment of diseases in the early stages is economically feasible. It is also obvious that for the timely detection of abnormalities it is necessary to ensure constant monitoring of indicators of the functional state of the organism. All this data can be transmitted remotely.

\subsection{Intellectualization of mobile medicine}

From 2000 years to the present day have marked the emergence of Smart healthcare - a health care service system that uses mobile medicine, the Internet of Things (IoT), the mobile Internet, for dynamic access to information, connection of people, materials and institutions related to health care, and in principle new active management of medical care. Smart healthcare can facilitate interaction between all participants in the field of health care, ensure that participants receive the services they need, help the parties to make informed decisions and promote the rational allocation of resources. In other words, intelligent health care is a new level of information design in the medical field [27].

Physicians can manage medical information through an integrated information platform that includes a laboratory information management system, picture archiving and communication system (PACS), an electronic medical record, and etc. More accurate surgery can be achieved with the help of surgical robots and mixed reality technology. From a hospital perspective, radio-frequency identification technology (RFID) can be used to manage human resources and the supply chain, using integrated management platforms to gather information and assist in decision-making [20, 24]. The use of mobile medical platforms can improve the experience of patients, from the point of view of research institutes, it is possible to use such methods as machine learning instead of manual drug screening and to find the necessary subjects using big data [22]. Using these smart healthcare technologies can effectively reduce the cost and risks of medical procedures, increase the efficiency of medical resources, promote exchange and cooperation in different regions, stimulate the development of telemedicine and self-care in health care, and ultimately make personalized medical services compatible. 


\subsection{Problems of minimizing scope of researches. Informativeness of symptoms}

One of the main problems in the use of mobile medicine is to minimize the number of features to be used in procedures for monitoring, assessing the severity of patients, differential diagnosis of their conditions and decision-making on possible intervention [4]. This problem is obviously clear, but its solution is very difficult and does not yet have a single solution. According to the research, the point is that there is never a complete certainty that the patient has identified all the changes in his organs and systems that may occur during observation [26]. It is clear that if such changes are detected during the providing of medical care in a medical institution, the correction of diagnostic and treatment procedures can be carried out almost without problems, but in cases of remote medicine, changing the treatment plan may be difficult [31].

Accordingly, the choice of features is of high differential significance and their aggregates in the dynamics of treatment of similar symptoms are the first important stage in the construction of mobile medicine systems [38].

Assessment of the significance of symptoms is often carried out using the characteristics of informativeness. It is important to note that these characteristics depend on the stage of use of clinical signs in the process of recognizing pathological processes [1, 39]. Indeed, at the first stage, when there are not many advantages of any of the diagnoses, a certain symptom can justify the strategy of further examination, ie to be very informative. In the further diagnosis of the pathological process, completely different clinical indicators may be important and provide a differential diagnosis [7]. Many methods of determining informativeness have been studied. They are usually divided into several groups - informational, probabilistic, conditionally probabilistic, spatial, graph-analytical and others. The Hartley function, or Hartley's amount of information, or Hartley's measure, is a logarithmic measure of information that determines the amount of information contained in a message [8].

More popular probabilistic algorithms are designed to solve problems, the exact solution of which is impossible or irrational, because the distribution of the variable in the sample differs from its distribution in the population. To determine and measure the impact of informativeness, we compare the design and model variances of the estimated parameters (as well as the estimated parameters themselves) in the logistics model under the assumption that the model that is postulated is true. For adequate modelling of data from informative samples, we consider two possibilities: to use the planned conclusion about the parameters of the model or the model conclusion.

\subsection{Probabilistic methods. Measures of Kullback and Shannon}

Kullback's method offers as an assessment of informativeness - a measure of the difference between the two classes. According to this method, informativeness is calculated by formula (1):

$$
J\left(x_{i}\right)=\sum 10 \lg \cdot P\left(x_{i j} / A_{1}\right) \cdot P\left(x_{i j} / A_{2}\right) \cdot 0.5\left[P\left(x_{i j} A_{1}\right)-P\left(x_{i j} / A_{2}\right)\right] \cdot i,
$$

$J\left(x_{i}\right)$ - informativeness of the feature, $P_{1}$ - the probability of hitting the feature in the first class $A_{1}$, $P_{2}$ - the probability of hitting the feature in the second class $A_{2}, j$ - feature range number $x_{i}$. It is clear that $J\left(x_{i}\right)$ is always $>0$. Hence, the value $J\left(x_{i}\right)$, being always positive, will reflect the absolute value of the contribution of this range in the approach to any correct diagnostic threshold.

In turn, Shannon's method - evaluates informativeness as a weighted average amount of information that falls on different gradations of the feature. Note that the measure of informativeness provides in this case the assessment of information regardless of its content [15].

Note that between the classical probabilistic estimates of the value of information to solve a particular problem and measures of information theory can be bridges. Thus, for the case of a uniform law of probability density distribution, Shannon's measure coincides with Hartley's measure. The main advantage of Shannon's formula is its abstractness from the semantic, qualitative and individual properties of the system. In contrast to the Hartley's formula, it takes into account the difference of different probability states - the formula has a statistical character, which makes this formula convenient for practical calculations $[40,16]$. Comparing the methods of determining the informativeness of the feature, it should be noted that the Shannon method allows to determine the 
informativeness of the feature involved in the recognition of any number of classes, and the sample size of observations for two recognizable classes may be different [10].

The relevance of the use of probabilistic algorithms is determined by the need for economical use of memory. They allow you to solve tasks that can be solved in other ways, but too expensive, which requires a lot of time and other resources, help to create cheaper and more predictable systems [27]. Summarizing this section, it should be emphasized that the use of methods of probability theory is more than acceptable, because despite the possibility of error, probabilistic algorithms should be used on big data, they contain a share of randomness, which can be quantified. This randomness can be analyzed and a reliable prediction of the behaviour of the algorithm can be obtained.

\subsection{Features of technologies of remote control of processes of rendering of mobile medical care}

Dozens of recent studies have addressed four main issues - the development and application of standards for receiving, transmitting and storing information when examining patients in a mobile format, ensuring the compatibility of devices used in mobile medicine, remote control of devices to obtain information about the patient's condition, and preservation of the patient's personal data [41]. Network technology that provides wireless data transmission with sensory devices located in the human body or around the human body is called the sensory network of the body (BSN). It can be used to monitor multiple vital metrics in real time, ensuring that data is stored through a base station on a remote server where it can be recorded and made available to medical personnel $[14,28]$. It is proven that this technology potentially provides significant benefits for the diagnosis and treatment of patients, as it minimizes limitations in daily life, which allows the patient to move freely, while providing continuous monitoring [18]. However, the wireless nature of network connections creates a number of problems for communication reliability [41].

The architecture of the combination of devices varies widely. But, most BSN systems proposed in the literature are based on a two-stage architecture, where sensitive devices transmit data over non-wired networks to a personal unit, which in turn provides data transfer to the base station [43, 44]. However, study [40] proposed an approach in which in a BSN system called Health Monitoring for All (HM4All), sensitive devices interact directly with the base station or with ZigBee routers. The latter are able to provide a route between devices and maintain communication. They are mainly used to extend the range of the ZigBee network. This approach has the advantage that the patient does not have to carry a personal unit. It also reduces the number of wireless connections between sensitive devices and the base station. It should be emphasized that, despite the large volume of research, there is, unfortunately, no common understanding regarding the standards of information transmission in mobile medicine [7, 38].

As you know, ZigBee is based on the CSMA protocol, which is used in two variations CSMA/CD (Carrier Sense Multiple Access with Collision Detection) - refers to the IEEE 802.3 standard, i. e. multiple access to the common transmitter environment in a local computer network with collision control. There are two variations of CSMA CA - collision avoidance (CA) and CSMA/CD (collision detection) refers to decentralized random (more precisely, quasi-random) methods. It is used both in conventional Ethernet networks and in high-speed networks (Fast Ethernet, Gigabit Ethernet). This is a common category of access control protocols in which stations listen to a channel before transmitting $[28,45]$.

Some versions of Ethernet use a variation of CSMA/CD, while wireless networks such as ZigBee and Wi-Fi use a second variation of CSMA-CA (Collision Avoidance), i. e. multiple access with carrier control and collision avoidance. It is a network protocol that uses a carrier wave listening circuit and the station that is about to start transmitting sends a special signal (congestion signal) and after a long wait of all stations that can send this signal, the station starts transmitting the data frame $[46,47]$. The node to which the data packet is to be transmitted performs the procedure of assessing the purity of the channel, i. e. listens to the noise in the transmission medium for a predetermined period of time. If the transmission medium is evaluated as clean, the node may transmit a data packet. Otherwise, if another transmission is performed, the node is «removed», i. e. waits a certain amount of time before starting the procedure of sending the packet again [12, 29]. 
Thus, in the first case, the stations stop their transmissions when a collision is detected, allowing the channel to be used again (to detect collisions, the stations listen for interference signals during their own transmissions). This approach is usually impractical for wireless networks because the interference signal is much weaker than the station's own signal. In this case, these networks rely on CA mechanisms [8]. However, in both cases, despite the use of the CSMA mechanism, the frequency of collisions tends to increase with increasing traffic load. In addition, CSMA-CA-based protocols are vulnerable to the problem of hidden nodes. In a CSMA-based network, a node can only transmit if the channel is idle. The hidden node problem also occurs when the carrier detection fails, and the node starts transmitting when another node has already occupied the channel. If both transmissions are within range of the receiver, a collision occurs, which significantly reduces the reliability of communication $[9,18]$.

The lower levels of the ZigBee protocol are defined by the IEEE 802.15.4 standard, which was widely accepted by the community of wireless sensor networks [44]. ZigBee is successfully used in several wireless sensor network applications, which typically generate event-based traffic and low data rates. Currently, it is also the most widely used protocol in BSN applications, the latter usually generate periodic and often intensive data traffic. However, IEEE 802.15.4 does not provide a specific mechanism for preventing the problem of hidden nodes, which has prompted some authors to consider specific scenarios and suggest strategies to mitigate it. The three most notable of these are related to the grouping of nodes that have two-way communication with each other [40]. However, these strategies require modifications to the source protocol and consider beacon-enabled networks consisting of static nodes, which is not the scenario discussed in this paper. Given the peculiarities of BSN traffic, it is necessary to evaluate the suitability of ZigBee for BSN applications [12].

The task of wireless networks WBAN (Wireless Body Area Network) is to maintain various medical applications, such as monitoring the condition of the body. We emphasize that there are two types of installation of sensor nodes: wearable devices (or can be in close proximity to the patient), and implantable devices [48].

However, using WBAN causes a lot of problems. They are related to the need to use extremely low power consumption, light weight, the ability to implant sensors, maintain security and confidentiality, ensure reliable transmission of vital data from the patient, the ability to call an ambulance, connect through disparate networks in real time, low complexity, proper use of standards, ensuring interoperability, low cost, ensuring high quality service, etc. [36]. Therefore, it is clear that with so many problems of high-quality and practical systems for monitoring the state of the organism as of today there are few [27]. Nevertheless, the study [11] discussed the possibilities of different types of intelligent medical sensory devices that can be placed on the human body. Physiological information comes from sensory devices and is processed in a data bank.

One of the most important issues in the implementation of WBAN is the design of routing structures and protocols. Routing protocols can be divided into two broad categories:

1) flat routing protocols, where each sensor node in the WBAN plays the same role;

2) hierarchical/cluster routing protocols, where different sensor nodes can play different roles.

Using the routing method, it is possible to easily measure the technological characteristics, in particular, how many packets will pass along this route from the sensor nodes. Five routing strategies were analyzed in $[39,40]$ : thermal routing protocols, cluster routing protocols, cross-level routing protocols, QoS-previously used routing protocols, and routing protocols with latency support.

The article [13] emphasizes the advantages of the routing method in WBAN. The bandwidth requirements for WBAN are relatively low. WBAN provides flexible data rates from 10 kilobits per second (Kbps) to 10 megabits per second (Mbps). Each sensor unit can operate at a speed of $250 \mathrm{Kbps}$ through the mechanism of cyclic mode. An efficient compression algorithm is required to transmit medical data, and reliability is considered to be another key factor $[6,49]$. WBAN uses licensed wireless medical telemetry services (WMTS) for the medical telemetry system, unlicensed ISM band (2.4-2.4835 GHz) and ultra-wideband (UWB) and medical implanted communication services (MICS) for data transmission. Other technologies are also used, such as wireless internet (IEEE 802.11/a standards, c, g), Bluetooth (IEEE 802.15.1 standard), ZigBee (IEEE 802.15.4 standard), etc. The WBAN transmission bandwidth requirement is $1.2 \mathrm{MHz}$. In terms of power 
consumption, UWB provides better performance than Bluetooth and ZigBee. UWB provides a highly integrated low-cost solution for modern, high-speed, short-distance communication. Wireless Broadband (WiBro) (IEEE 802.16 e) is a new type of mobile WiMax that supports real-time medical data transmission without space and time constraints [40].

Note that remote control of devices is acute both in the tasks of «pure» mobile medicine and in the format of a new approach, called the «Internet of Things» (IoT) and machine-to-machine (M2M) interaction. Bluetooth Low Energy (BLE) and ZigBee are most commonly used in IoT. The devices operate at frequencies of $2.4 \mathrm{GHz}$. When designing BAN-networks, the main attention is paid to energy efficiency, which implies low energy consumption by nodes. Also, these networks support only the star topology, however, with a high degree of scalability.

BANs use several sensor and/or actuators of nodes that communicate with a network controller (BAN Network Controller, BNC), implemented, for example, in the form of a phone or laptop [40]. The received information can be transferred to the user's device, and the use of a specially developed application will allow to calculate human activity, change health indicators and, if necessary, will give recommendations for the successful achievement of the user's goal. In the possibility of modelling and visualization of WBAN systems for telemedicine is analyzed, which may constitute another future era of sensor networks [1, 41]. According to the authors, the goals of the system could be the collection and processing of vital information about patients; fast and secure data transfer; accurate and fast monitoring and diagnosis of the body. Fig. 1 shows the proposed structure of the network telemedicine system with sensors on the surface of the body. It consists of three stages based on the ease of use of standards and technologies.

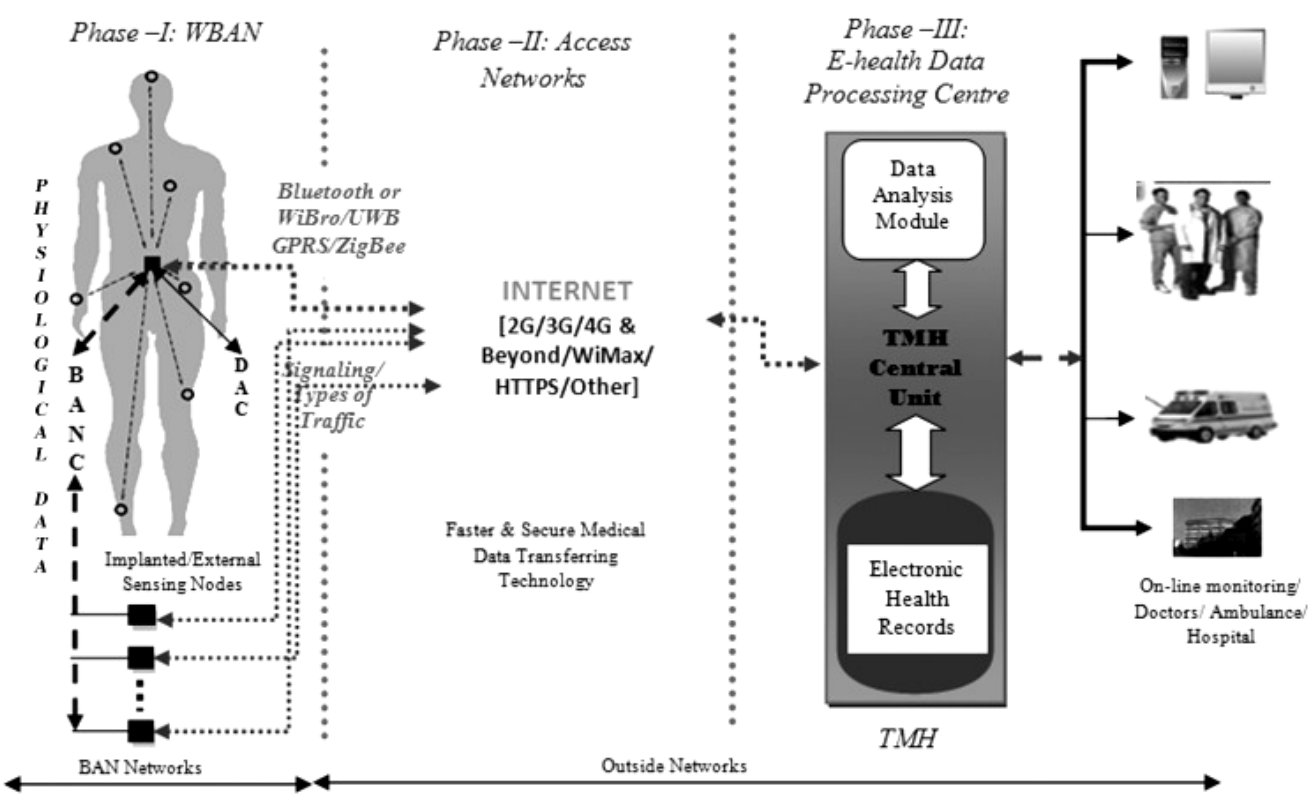

Fig. 1. Telemedicine system with remote monitoring of the object: BAN - network of the body area; BANC - coordinator of the body area network; DAC - data collection center; $\mathrm{TMH}$ - telemedicine hub; WBAN - wireless network of the body area

In the first stage, the authors proposed a WBAN, consisting of several external and internal sensor nodes, each of which receives certain physiological data from the human body and interacts with the main node, known as BANC. It is believed that the possibility of «star» topology is quite sufficient in WBAN (network coverage of 2 to 5 meters), where BANC is located centrally and each sensor is directly connected to the main node. As a rule, several BANCs are required to continuously obtain clinical data from the source nodes. Based on the critical condition of patients, the data is processed and transmitted to medical experts, as well as stored on a specialized medical server, which can be used for statistical modelling, compression, privacy, optimal path design and process planning. For the purposes of collecting images and data, an image archiving system has been proposed [40, 41]. 


\subsection{Features of problem statement in e-health}

Traditional systems collect data using sensors attached to the body. But these systems create difficulties in terms of mobility for the patient and his activities in everyday life. Because such devices affect patient comfort, sensitive physiological data are affected. Thus, the indications may not represent the actual disease of the patient, but rather indicators of the discomfort he feels during physiological examinations [13].

It has already been noted that the informational content of the research depends on its place in the diagnostic process. This dependence «acts» due to the probability of diagnoses calculated on the basis of previous studies [14]. These probabilities are a priori for calculating the possibilities of diagnoses after receiving the results of the next examination. Therefore, it is desirable to obtain such estimates of informational content, which would not depend on the a priori distribution of probabilities of pathological processes (condition of patients) [21, 50]. The author has previously proposed the use of risk indicators of the condition (or transition to another condition with higher risk characteristics) as a universal method of determining the informational content of symptoms.

Hence the basic principle of construction of the diagnostic process: the decision is made for which the average risk associated with its error will be minimal. The next steps are to determine the informational content of the signs that change continuously during the diagnostic process, for example, during the monitoring of the patient's condition in e-health. In this case, to assess the informational content of the signs for the transition between the two states of the patient, it is proposed to use the formulas of Kullback and Shannon [6, 14, 41]. The calculation of informational content according to Shannon is carried out as a weighted average amount of information per different gradations of the feature. Accordingly, it receives an estimate of informational content as a normalized value, which varies from 0 to 1 . Therefore, the informational content of the feature determined by the Shannon method can be said in digital representation from 1 (highest informational content to 0 - lowest).

Interestingly, in most studies there are no recommendations or justifications in which cases should apply one or another measure of informational content.

\section{Discussion}

\subsection{Key points of systematic research}

Trials of support from healthcare providers show some promising results in terms of clinical management, appropriate testing, referral, screening, diagnosis, treatment and triage. However, the studies included in our review had a high or unclear risk of bias. In particular, only one of trials clearly reported that the distribution was hidden, and if there is no concealment of the distribution, the reported results may overestimate the effects [7, 51]. To date, no trials have reported the impact of mobile-based clinical diagnosis and management on objective health outcomes. Most studies to support healthcare providers in clinical diagnosis and treatment have used PDAs and specialized application software functions. Although handheld computers are no longer widely used, customizable features in application software are now available on smartphones or tablets. Mobile-based interventions may not be appropriate for some clinical processes.

The data available for making a clinical diagnosis or calculating early warning scores can be reduced, and the time required for medical processes can be increased. There was no clear evidence of the benefits of mobile-based educational activities for healthcare providers [7]. With regard to interventions using mobile technologies for transmission of visual data, the time for diagnosis, transmission of ECG or diagnostic errors has increased. Two trials using photographs taken with a mobile phone reduced the accuracy of the diagnosis of fractures, skin ecchymosis and the possibility of re-implantation compared to the gold standard. However, the use of such technologies may be more relevant in conditions where the gold standard is not available. In addition, the quality of photos on mobile phones has improved since the completion of these studies.

Mobile-based diagnostic and management support may be most relevant to healthcare providers in developing countries, where mobile phones have the potential to provide evidence-based clinical support and guidance to telecommuting healthcare providers and in circumstances where a senior healthcare professional support staff or other infrastructure not available [52]. One high-quality trial reported increased adherence of health care workers in Kenya to treatment 
recommendations for malaria [53]; however, data from controlled trials to date have come mainly from high-income countries, where the «standard care» control group can be very different from the «standard care» available in low- or middle-income countries.

\subsection{Advantages and disadvantages}

To the best of our knowledge, this is the first comprehensive systematic review of trials of all mobile technology interventions delivered to healthcare providers and healthcare service support to improve healthcare or healthcare delivery. The review expands and updates the results of previous systematic reviews that focus on specific devices and/or specific functions and/or specific health topics [11, 51]. We found more than double the number of tests of training activities and tests of PDA programs identified in previous reviews $[11,25]$. The results of our review are consistent with the findings of Krishna et al. and car texting can reduce missed appointments [44, 51].

Our systematic review was extensive. We pooled results only when the intervention function (e.g., SMS messages), the trial objective, and the results used in the trials were the same. It summarizes the findings regarding clinical diagnosis and treatment and educational interventions [54]. It was impractical to combine these results because the measures were aimed at different diseases and outcomes. In addition, it is likely that there are important differences in the content of the interventions of these interventions (such as the behavioural change methods used), even in those that use the same mobile technology functions (for example, application software). It was not possible to investigate how the different components of the intervention influenced the results, as the components of the intervention were not described consistently or in detail in the authors' works [7]. It was not possible to investigate how disease components of the intervention and outcomes influenced outcomes.

We were unable to statistically investigate the factors that contribute to heterogeneity because there have been few studies of similar interventions that reported the same results, resulting in limited scope for such analyzes. It was not possible to statistically investigate the mechanism of action of the interventions because there were too few similar interventions that reported the same results [7]. In addition, the authors of the descriptions of the interventions were not detailed enough to allow the study of the mechanisms of action. Research into the cost-effectiveness of interventions with modest benefits, such as reminder to prescribe, was outside the scope of this review.

\subsection{Challenges and Prospects for Future Research}

High quality trials are needed to establish the impact of clinical diagnosis and management support (e.g. protocols/decision support systems) on clinical outcomes using customizable mobile phone application software functions. The impact of such support on the management of various diseases and on objective disease outcomes should be assessed. It is imperative that future trials of mobile clinical decision support tools, guidelines and protocols be conducted in low- and middle-income countries. Many of the interventions evaluated to date are low-intensity, single-component interventions [7]. The consequences of more intensive interventions using multicomponent mobile technologies should be evaluated. Authors should describe in detail the components of future interventions so that the mechanisms of action and the impact of different components on outcome can be examined.

Trials should evaluate the impact of using photo or video features to support healthcare providers versus standard care (where gold standard options are not available) [22, 51]. As the technology capabilities of mobile phones improve, for example in terms of photographic quality, further testing may be required on the impact of mobile photography on diagnostic accuracy [55]. Further research should evaluate the impact and cost-effectiveness of mobile technologies to increase the speed of communication between clinicians and patients, such as test results.

Interventions that combine elements of mobile technology with other treatments, such as clinical counselling combined with text messaging, should be systematically reviewed.

\section{Conclusions}

1. There was proposed to use risk indicators of predicted conditions as a universal method for determining the informational content of symptoms in mobile monitoring of patients. 
2. Taking into account that the informational content of the patient's condition is constantly changing, for online diagnosis of the patient's body during remote observation of the patient it is recommended to use the function of informative symptoms, as well as use a set of approaches for comprehensive assessment. It is proposed to use the strategy of diagnosis and treatment using probabilistic algorithms based on the values of the risk of complications of the pathological process, as well as the formulas of Kullback and Shannon to determine individual trends in the pathological process in the patient.

\section{Conflict of interests}

The authors declare that they have no conflicts of interest.

\section{Financing}

The study was performed without financial support.

\section{References}

[1] Boyce, B. (2017). An update on the validation of whole slide imaging systems following FDA approval of a system for a routine pathology diagnostic service in the United States. Biotechnic \& Histochemistry, 92 (6), 381-389. doi: http://doi.org/10.1080/ 10520295.2017.1355476

[2] Akmandor, A. O., Jha, N. K. (2017). Keep the Stress Away with SoDA: Stress Detection and Alleviation System. IEEE Transactions on Multi-Scale Computing Systems, 3 (4), 269-282. doi: http://doi.org/10.1109/tmscs.2017.2703613

[3] Free, C., Phillips, G., Watson, L., Galli, L., Felix, L., Edwards, P. et. al. (2013). The Effectiveness of Mobile-Health Technologies to Improve Health Care Service Delivery Processes: A Systematic Review and Meta-Analysis. PLoS Medicine, 10 (1), e1001363. doi: http://doi.org/10.1371/journal.pmed.1001363

[4] Denis, F., Basch, E., Septans, A.-L., Bennouna, J., Urban, T., Dueck, A. C., Letellier, C. (2019). Two-Year Survival Comparing Web-Based Symptom Monitoring vs Routine Surveillance Following Treatment for Lung Cancer. JAMA, 321 (3), $306-307$. doi: http://doi.org/10.1001/jama.2018.18085

[5] Gagnon, M.-P., Ngangue, P., Payne-Gagnon, J., Desmartis, M. (2015). m-Health adoption by healthcare professionals: a systematic review. Journal of the American Medical Informatics Association, 23 (1), 212-220. doi: http://doi.org/ 10.1093/jamia/ocv052

[6] Hajian, T. K. (2012) Methodological issues of confounding in analytical epidemiologic studies. Caspian Journal of Internal Medicine, 3 (3), 488-495.

[7] Jabour, A. M., Rehman, W., Idrees, S., Thanganadar, H., Hira, K., Alarifi, M. A. (2021). The Adoption of Mobile Health Applications Among University Students in Health Colleges. Journal of Multidisciplinary Healthcare, 14, $1267-1273$. doi: http://doi.org/10.2147/jmdh.s310539

[8] Kapustina, S. V., Kiryakova, O. V., Kapustina, A. V., Lapina, L. A., Stupina, A. A. (2015). The choice of informative features for assessing the severity of the disease. Modern problems of science and education, 2 (2), 55.

[9] Engel, H., Huang, J. J., Tsao, C. K., Lin, C.-Y., Chou, P.-Y., Brey, E. M. et. al. (2011). Remote real-time monitoring of free flaps via smartphone photography and $3 \mathrm{G}$ wireless internet: A prospective study evidencing diagnostic accuracy. Microsurgery, 31 (8), 589-595. doi: http://doi.org/10.1002/micr.20921

[10] Hajat, C. (2010). An Introduction to Epidemiology. Genetic Epidemiology, 27-39. doi: http://doi.org/10.1007/978-1-60327-416-6_3

[11] Kho, A., Henderson, L. E., Dressler, D. D., Kripalani, S. (2006). Use of handheld computers in medical education: A systematic review. Journal of General Internal Medicine, 21 (5), 531-537. doi: http://doi.org/10.1111/j.1525-1497.2006.00444.x

[12] Kachmar, V. O., Avramenko, V. I. (2011). The trends of the informational technologies' development in medicine. Transport Medicine of Ukraine, 3, 96-103.

[13] Griffin, J., Treanor, D. (2017). Digital pathology in clinical use: where are we now and what is holding us back? Histopathology, 70 (1), 134-145. doi: http://doi.org/10.1111/his.12993

[14] Kamel Boulos, M. N., Wilson, J. T., Clauson, K. A. (2018). Geospatial blockchain: promises, challenges, and scenarios in health and healthcare. International Journal of Health Geographics, 17 (1). doi: http://doi.org/10.1186/s12942-018-0144-x

[15] Geller, N. L., Kim, D.-Y., Tian, X. (2016). Smart Technology in Lung Disease Clinical Trials. Chest, 149 (1), 22-26. doi: http:// doi.org/10.1378/chest.15-1314

[16] Florczak, B., Scheurich, A., Croghan, J., Sheridan, P. Jr., Kurtz, D., McGill, W., McClain, B. (2012). An Observational Study to Assess an Electronic Point-of - Care Wound Documentation and Reporting System Regarding User Satisfaction and Potential for Improved Care. Ostomy Wound Manage, 58, 46-51. 
[17] Bakkar, N., Kovalik, T., Lorenzini, I., Spangler, S., Lacoste, A., Sponaugle, K. et. al. (2017). Artificial intelligence in neurodegenerative disease research: use of IBM Watson to identify additional RNA-binding proteins altered in amyotrophic lateral sclerosis. Acta Neuropathologica, 135 (2), 227-247. doi: http://doi.org/10.1007/s00401-017-1785-8

[18] Chen, M., Gonzalez, S., Vasilakos, A., Cao, H., Leung, V. C. M. (2010). Body Area Networks: A Survey. Mobile Networks and Applications, 16 (2), 171-193. doi: http://doi.org/10.1007/s11036-010-0260-8

[19] Dhar, J., Ranganathan, A. (2015). Machine learning capabilities in medical diagnosis applications: computational results for hepatitis disease. International Journal of Biomedical Engineering and Technology, 17 (4), 330-340. doi: http://doi.org/10.1504/ ijbet.2015.069398

[20] Gong, F. F., Sun, X. Z., Lin, J., Gu, X. D. (2013). Primary exploration in establishment of China's intelligent medical treatment. Mod. Hos. Manag, 11 (2).

[21] Esteva, A., Kuprel, B., Novoa, R. A., Ko, J., Swetter, S. M., Blau, H. M., Thrun, S. (2017). Dermatologist-level classification of skin cancer with deep neural networks. Nature, 542 (7639), 115-118. doi: http://doi.org/10.1038/nature21056

[22] Hsieh, C.-H., Tsai, H.-H., Yin, J.-W., Chen, C.-Y., Yang, J. C.-S., Jeng, S.-F. (2004). Teleconsultation with the Mobile Camera-Phone in Digital Soft-Tissue Injury: A Feasibility Study. Plastic and Reconstructive Surgery, 114 (7), $1776-1782$. doi: http://doi.org/10.1097/01.prs.0000142402.07896.21

[23] Dunn, J., Runge, R., Snyder, M. (2018). Wearables and the medical revolution. Personalized Medicine, 15 (5), $429-448$. doi: http://doi.org/10.2217/pme-2018-0044

[24] Eysenbach, G., Diepgen, T. L. (2001). The role of e-health and consumer health informatics for evidence-based patient choice in the 21st century. Clinics in Dermatology, 19 (1), 11-17. doi: http://doi.org/10.1016/s0738-081x(00)00202-9

[25] Basch, E., Deal, A. M., Dueck, A. C., Scher, H. I., Kris, M. G., Hudis, C., Schrag, D. (2017). Overall Survival Results of a Trial Assessing Patient-Reported Outcomes for Symptom Monitoring During Routine Cancer Treatment. JAMA, 318 (2), $197-198$. doi: http://doi.org/10.1001/jama.2017.7156

[26] Pantanowitz, L., Guo, H., Birsa, J., Farahani, N., Hartman, D., Piccoli, A. et. al. (2016). Digital pathology and anatomic pathology laboratory information system integration to support digital pathology sign-out. Journal of Pathology Informatics, 7 (1), 23. doi: http://doi.org/10.4103/2153-3539.181767

[27] Hande, A., Polk, T., Walker, W., Bhatia, D. (2006). Self-Powered Wireless Sensor Networks for Remote Patient Monitoring in Hospitals. Sensors, 6 (9), 1102-1117. doi: http://doi.org/10.3390/s6091102

[28] Greenland, S., Morgenstern, H. (2001). Confounding in Health Research. Annual Review of Public Health, 22 (1), $189-212$. doi: http://doi.org/10.1146/annurev.publhealth.22.1.189

[29] Buratti, C., Conti, A., Dardari, D., Verdone, R. (2009). An Overview on Wireless Sensor Networks Technology and Evolution. Sensors, 9 (9), 6869-6896. doi: http://doi.org/10.3390/s90906869

[30] Demirkan, H. (2013). A Smart Healthcare Systems Framework. IT Professional, 15 (5), 38-45. doi: http://doi.org/10.1109/ mitp.2013.35

[31] Farahani, B., Firouzi, F., Chang, V., Badaroglu, M., Constant, N., Mankodiya, K. (2018). Towards fog-driven IoT eHealth: Promises and challenges of IoT in medicine and healthcare. Future Generation Computer Systems, 78 (2), $659-676$. doi: http://doi.org/10.1016/j.future.2017.04.036

[32] Álvarez López, Y., Franssen, J., Álvarez Narciandi, G., Pagnozzi, J., González-Pinto Arrillaga, I., Las-Heras Andrés, F. (2018). RFID Technology for Management and Tracking: e-Health Applications. Sensors, 18 (8), 2663. doi: http://doi.org/10.3390/s18082663

[33] Andreu-Perez, J., Leff, D. R., Ip, H. M. D., Yang, G.-Z. (2015). From Wearable Sensors to Smart Implants-Toward Pervasive and Personalized Healthcare. IEEE Transactions on Biomedical Engineering, 62 (12), 2750-2762. doi: http:/doi.org/10.1109/tbme.2015.2422751

[34] Garvin, W. (2012). The Legal Perspective of mHealth in the United States. Journal of Mobile Technology in Medicine, 1 (4), 42-45. doi: http://doi.org/10.7309/jmtm.82

[35] Estrin, D., Sim, I. (2010). Open mHealth Architecture: An Engine for Health Care Innovation. Science, 330 (6005), 759-760. doi: http://doi.org/10.1126/science.1196187

[36] Willard-Grace, R., DeVore, D., Chen, E. H., Hessler, D., Bodenheimer, T., Thom, D. H. (2013). The effectiveness of medical assistant health coaching for low-income patients with uncontrolled diabetes, hypertension, and hyperlipidemia: protocol for a randomized controlled trial and baseline characteristics of the study population. BMC Family Practice, 14 (1).

[37] doi: http://doi.org/10.1186/1471-2296-14-27

[38] Janes, H., Pepe, M. S. (2008). Adjusting for Covariates in Studies of Diagnostic, Screening, or Prognostic Markers: An Old Concept in a New Setting. American Journal of Epidemiology, 168 (1), 89-97. doi: http://doi.org/10.1093/aje/kwn099

[39] Denis, F., Yossi, S., Septans, A.-L., Charron, A., Voog, E., Dupuis, O. et. al. (2017). Improving Survival in Patients Treated for a Lung Cancer Using Self-Evaluated Symptoms Reported Through a Web Application. American Journal of Clinical Oncology, 40 (5), 464-469. doi: http://doi.org/10.1097/coc.0000000000000189 
[40] Ben Elhadj, H., Chaari, L., Kamoun, L. (2012). A Survey of Routing Protocols in Wireless Body Area Networks for Healthcare Applications. International Journal of E-Health and Medical Communications, 3 (2), 1-18. doi: http://doi.org/10.4018/ jehmc. 2012040101

[41] Fernandez-Lopez, H., Afonso, J. A., Correia, J. H., Simoes, R. (2014). Remote Patient Monitoring Based on ZigBee: Lessons from a Real-World Deployment. Telemedicine and e-Health, 20 (1), 47-54. doi: http://doi.org/10.1089/tmj.2013.0059

[42] Blair, A., Stewart, P., Lubin, J. H., Forastiere, F. (2007). Methodological issues regarding confounding and exposure misclassification in epidemiological studies of occupational exposures. American Journal of Industrial Medicine, 50 (3), $199-207$. doi: http://doi.org/10.1002/ajim.20281

[43] Austin, P. C. (2011). An Introduction to Propensity Score Methods for Reducing the Effects of Confounding in Observational Studies. Multivariate Behavioral Research, 46 (3), 399-424. doi: http://doi.org/10.1080/00273171.2011.568786

[44] Barakah, D. M., Ahmad-Uddin, M. (2012). A survey of challenges and applications of wireless body area network (WBAN) and role of a virtual doctor server in existing architecture. 3rd International Conference on Intelligent Systems Modelling and Simulation. Piscataway: IEEE, 214-219. doi: http://doi.org/10.1109/isms.2012.108

[45] Car, J., Gurol-Urganci, I., de Jongh, T., Vodopivec-Jamsek, V., Atun, R. (2012). Mobile phone messaging reminders for attendance at healthcare appointments. Cochrane Database of Systematic Reviews, 7, CD007458. doi: http://doi.org/10.1002/ 14651858.cd007458.pub2

[46] Chen, Q., Lu, Y. (2018). Construction and application effect evaluation of integrated management platform of intelligent hospital based on big data analysis. China Medical Herald, 15 (35), 161-164.

[47] Chakraborty, C., Gupta, B., Ghosh, S. K. (2013). A Review on Telemedicine-Based WBAN Framework for Patient Monitoring. Telemedicine and e-Health, 19 (8), 619-626. doi: http://doi.org/10.1089/tmj.2012.0215

[48] Curtis, D. W., Pino, E. J., Bailey, J. M., Shih, E. I., Waterman, J., Vinterbo, S. A. et. al. (2008). SMART-An Integrated Wireless System for Monitoring Unattended Patients. Journal of the American Medical Informatics Association, 15 (1), $44-53$. doi: http://doi.org/10.1197/jamia.m2016

[49] Ali, M., Saif, U., Dunkels, A., Voigt, T., Römer, K., Langendoen, K. et. al. (2006). Medium access control issues in sensor networks. ACM SIGCOMM Computer Communication Review, 36 (2), 33-36. doi: http://doi.org/10.1145/1129582.1129592

[50] Bastawrous, A., Leak, C., Howard, F., Kumar, V. (2012). Validation of Near Eye Tool for Refractive Assessment (NETRA) Pilot Study. Journal of Mobile Technology in Medicine, 1 (3), 6-16. doi: http://doi.org/10.7309/jmtm.17

[51] Chih-Chung Huang, Po-Yang Lee, Pay-Yu Chen, Ting-Yu Liu. (2012). Design and implementation of a smartphone-based portable ultrasound pulsed-wave doppler device for blood flow measurement. IEEE Transactions on Ultrasonics, Ferroelectrics and Frequency Control, 59 (1), 182-188. doi: http://doi.org/10.1109/tuffc.2012.2171

[52] Krishna, S., Boren, S. A., Balas, E. A. (2009). Healthcare via Cell Phones: A Systematic Review. Telemedicine and e-Health, 15 (3), 231-240. doi: http://doi.org/10.1089/tmj.2008.0099

[53] Mechael, P. N., Batavia, H., Kaonga, N., Searle, S., Kwan, A. et. al. (2010). Barriers and Gaps Affecting mHealth in Low and Middle Income Countries: Policy White Paper. Center for Global Health and Economic Development, Earth Institute, Columbia University, 79.

[54] Zurovac, D., Sudoi, R. K., Akhwale, W. S., Ndiritu, M., Hamer, D. H., et. al. (2011). The effect of mobile phone text-message reminders on Kenyan health workers' adherence to malaria treatment guidelines: a cluster randomised trial. Lancet, 378, 795-803. doi: http://doi.org/10.1016/s0140-6736(11)60783-6

[55] Lindquist, A. M., Johansson, P. E., Petersson, G. I., Saveman, B.-I., Nilsson, G. C. (2008). The Use of the Personal Digital Assistant (PDA) Among Personnel and Students in Health Care: A Review. Journal of Medical Internet Research, 10 (4), e31. doi: http://doi.org/10.2196/jmir.1038

[56] Boulos, M., Wheeler, S., Tavares, C., Jones, R. (2011). How smartphones are changing the face of mobile and participatory healthcare: an overview, with example from eCAALYX. BioMedical Engineering OnLine, 10 (1), 24. doi: http://doi.org/ $10.1186 / 1475-925 x-10-24$

Received date 03.08.2021

(C) The Author(s) 2021

Accepted date 06.09.2021

Published date 30.09.2021

This is an open access article under the Creative Commons CC BY license

How to cite: Shevchenko, Y. (2021). Determination of the informational content of symptoms in the dynamic processes of assessing the patient's condition in e-health (literature review). EUREKA: Health Sciences, 5, 47-60. doi: http://doi.org/ $10.21303 / 2504-5679.2021 .001976$ 